\title{
Possible aetiology of haemorrhagic shock and encephalopathy syndrome in the Negev area of Israel
}

\author{
Shaul Sofer, Baruch Yerushalmi, Eliezer Shahak, Tamar Berenstein, Hanna Schulman
}

\begin{abstract}
A retrospective study was performed for all patients diagnosed with haemorrhagic shock and encephalopathy syndrome (HSES) over an 11 year period (1984-94). Soroka University Medical Centre is the only medical facility in the southern Negev region of Israel serving a population of about 400000 residents, consisting primarily of two ethnic populations, Jews and Bedouins.
\end{abstract}

Twenty patients, 17 Bedouin and three Jews, were diagnosed with HSES. The annual incidence of HSES for infants under the age of 1 year was 5:10 000 for Bedouins and 0.6:10 000 for Jews. Patients ranged in age from 6 to 32 weeks and arrived at the hospital late at night or early morning (2.00 am to $11.00 \mathrm{am}$ ), during the winter or early spring (November to April). All were healthy before admission, with short prodromal symptoms of upper respiratory tract or gastrointestinal infection noted in 10 cases. Most infants had markedly high body temperature on arrival. A history of overwrapping and/or excessive heating was obtained in four of 20 infants. Bacteriological and virological cultures were negative in all infants. One infant died and neurological sequelae were observed in all survivors.

The high prevalence of hyperpyrexia during sleep in the presence of negative microbiological results with no evidence of excessive heating, and the high incidence of HSES among a closed and culturally isolated society known to have a high incidence of congenital malformations, may support previous assumptions that HSES results from hyperpyrexia, originating in most cases from a 'physiological' heat induced trigger, which starts and peaks during the night in previously healthy infants who are genetically susceptible.

(Arch Dis Child 1996;75:332-334)

Keywords: haemorrhagic shock, encephalopathy, hyperthermia, Bedouins.

Levin et al first described haemorrhagic shock and encephalopathy syndrome (HSES) in 1983 as acute shock, encephalopathy, bleeding coagulopathy, and hepatorenal syndrome in previously healthy infants. ${ }^{1}$ This syndrome has been reported worldwide. ${ }^{2-8}$ The outcome is usually poor, and the aetiology is as yet unknown. In this report, we describe 20 cases of HSES in infants over an 11 year period in the Negev, the southern region of Israel.

\section{Subjects and methods}

Soroka University Medical Centre is the only hospital in the southern Negev area of Israel, serving a population of about 400000 of which approximately $75 \%$ are Jews and $25 \%$ Bedouins. We reviewed medical records of all patients admitted to the paediatric intensive care unit (PICU) between 1 January 1984 and 31 December 311994 with shock, encephalopathy, and bleeding coagulopathy. HSES, defined by Bacon and Hall, ${ }^{9}$ includes the presence of all of the following clinical and laboratory findings: encephalopathy, shock, disseminated intravascular coagulation, diarrhoea (may be bloody), falling haemoglobin concentration and platelet count, acidosis, raised hepatocellular enzymes, renal function impairment, and negative cultures of blood and cerebrospinal fluid. Twenty of our patients met these criteria and were included in the study.

Hospital delivery room records and official Ministry of Health statistical reports were used in order to calculate incidence of HSES among Jewish and Bedouin populations.

\section{Results}

GENERAL AND EPIDEMIOLOGICAL FEATURES

The infants ranged in age from 6 to 32 weeks (mean 15.5; median 14 weeks). Sixteen were boys and four girls. Seventeen infants were Bedouins with consanguinity between parents in nine cases; three infants were Jews. The annual incidence of HSES, for infants under the age of 1 year, was 5:10 000 for Bedouins and 0.6:10 000 for Jews.

All patients arrived at the emergency room during the winter season or early spring (fig 1), late at night or in the early morning hours that peaked between 5.00 am and 9.00 am (fig 2). The distribution of cases among years was similar and varied from none to three cases annually.

All infants had been previously healthy with a normal developmental course. One infant had been born prematurely ( 28 weeks' gestation). Ten infants had mild prodromal illness with respiratory $(n=6)$ or gastrointestinal $(n=$ 4) symptoms. The remaining 10 infants had been well until the onset of the main illness, 
which in all cases was abrupt and catastrophic. A history of overwrapping and excessive heating was obtained for four infants and for 16 infants this was strongly denied by the parents. Six of the 20 patients have been previously reported. ${ }^{410} 11$

CLINICAL AND LABORATORY FEATURES

All patients exhibited classical clinical and laboratory features of HSES. $^{1-13}$ Profound shock, coma with convulsions, and respiratory disturbances were the main features on arrival. Active bleeding and/or disturbed coagulation tests with falling haemoglobin concentrations and thrombocytopenia was noted in every case. All infants developed diarrhoea shortly after arrival (eight had bloody diarrhoea). Raised urea and creatinine was noted in all cases on arrival while raised liver enzymes were found in 19 cases (not measured in one case) at 24 to 48 hours after arrival.

Seventeen infants had raised rectal temperature on arrival at the emergency room. In 11 of 17 infants, temperature ranged between $40^{\circ} \mathrm{C}$ and $42^{\circ} \mathrm{C}$. However, three infants were slightly hypothermic on arrival (rectal temperatures $34^{\circ} \mathrm{C}$ in two infants and $35^{\circ} \mathrm{C}$ in one). These three infants were reported by the parents as feeling hot before arrival. In two of three cases, the infants were taken to a private paediatrician in the city of Beer-Sheva before being admitted to hospital. Rectal temperatures of $37.5^{\circ} \mathrm{C}$ and $38^{\circ} \mathrm{C}$ were recorded at that time by the physician. All infants had lower rectal temperature on arrival at the PICU compared with rectal temperatures on arrival at the emergency room. The difference in mean rectal tempera-

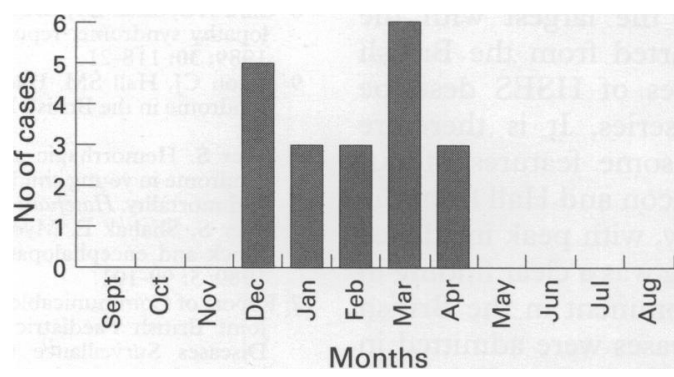

Figure 1 Distribution of HSES admissions, 1984-94.

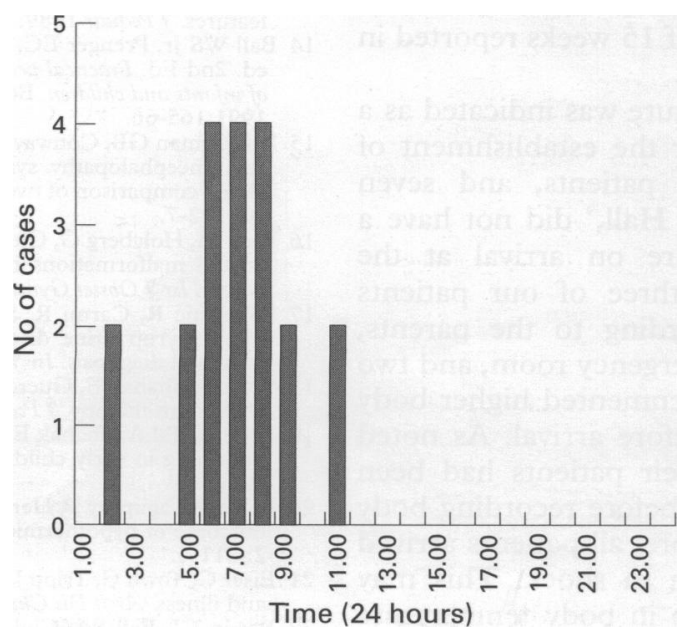

Figure 2 Time of arrival to emergency room of infants with HSES, 1984-94. ture (within one hour) from emergency room and PICU arrival was $3.38^{\circ} \mathrm{C}$.

Other noteworthy features on arrival were: hypoglycaemia (glucose $0-2.1 \mathrm{mmol} / \mathrm{l}$ ) in 13 of 20 infants, and hypernatraemia (sodium $>145 \mathrm{mmol} / \mathrm{l}$ ) in seven infants. Brain computed tomography was normal in six infants and showed ischaemic infarcts in 10 infants. Cerebral bleeding was found in three cases in addition to ischaemia. Three infants showed the classic picture, defined radiologically as 'reversal sign' consisting of loss of grey-white matter differentiation due to diffuse brain oedema and necrosis. ${ }^{14}$ Bacteriological cultures of blood, cerebrospinal fluid, urine, and stool and virological tests were negative in all cases.

Treatment included fluid resuscitation and inotropic drugs, mechanical ventilation, blood and blood products, anticonvulsive therapy, and other symptomatic treatment. One infant died and 19 infants survived; two had moderate psychomotor retardation and neurological handicap and 17 had severe impairment.

\section{Discussion}

Although first reported in $1983,{ }^{1}$ HSES has not been recognised as a distinct clinicopathological entity. ${ }^{915}$ The incidence and aetiopathogenesis remains unclear. The main aetiological factors suggested in the literature are: infectious micro-organisms or toxins; environmental factors (overwrapping, excessive heating); metabolic disorders (protease inhibitor deficiency; stress protein deficiency; malignant hyperthermia); and immaturity of the thermoregulatory centre. 13491315

Despite an extensive search, no causative micro-organisms or toxins were found in our patients or identified by other authors. Heatstroke due to overwrapping, swaddling, or excessive heating could not be documented in most of our patients or in those reported by others. ${ }^{1-391213}$ The assumption that poor (immature) thermoregulation and/or thermoregulatory imbalance may result in hyperpyrexia after being exposed to (common) physiological stress (fever due to viral infection or warm environmental area), ${ }^{3415}$ cannot be excluded. Metabolic disorders with deficiencies of protease inhibitors and abnormalities in stress protein production were first suggested by Levin et al who documented raised serum trypsin coupled with low $\alpha_{1}$-antitrypsin values in patients with HSES. ${ }^{13}$ Six of 19 first degree relatives of HSES patients in the study of Levin et al had a non-M phenotype for $\alpha_{1}$ antitrypsin. It was postulated that patients with HSES may have an inherited defect in synthesis and secretion of stress proteins and that a physiological stress may trigger the release of trypsin, elastin, and other proteases, which, left unopposed by protease inhibitors, may lead to a massive inflammatory response manifesting as HSES. Malignant hyperpyrexia, a familial disorder, in which the genetic factors remain unclear, was also proposed as a possible metabolic defect. ${ }^{315}$

In our study, we have two distinct ethnic populations cohabiting in the same geographical area, and served by the same hospital. The 
Bedouins represent a closed and culturally isolated society where a marriage within the family is customary rather than exceptional. As a result, there is an unusually high incidence of congenital malformations due to both autosomal recessive and multifactorial polygenic genetic disorders. ${ }^{16}{ }^{17}$ Thus, we calculated the incidence of HSES in our area and compared it with the rate between Bedouins and Jews. The high incidence of HSES among Bedouins may support the assumption of a genetic predisposition, including inborn error of (protein) metabolism and/or disorder (immaturity) of the thermoregulatory centre. The high prevalence of documented consanguinity in Bedouin families (nine of 17 cases) may support this assumption.

It should be noted, however, that environmental manipulations cannot be excluded as several environmental, non-genetic aetiologies such as traumatic accidents and burns (unpublished data), envenomation, ${ }^{18}$ intoxication, ${ }^{19}$ and neonatal hypothermia ${ }^{20}$ are more prevalent among Bedouins than Jews. This however cannot explain the difference in HSES incidence. Differences in behaviour regarding dress and care of infants in health and illness (type of clothing, swaddling, bundling, ambient temperature) between the two populations could have explained the trend toward hyperthermia in Bedouin infants. This issue however has not been thoroughly investigated. In the Exeter area (UK), it has been found that less educated mothers kept their infants in warmer rooms and were less likely to treat high temperature than infants of more educated mothers. ${ }^{21}$

To the best of our knowledge, our series of patients with HSES is the largest with the exception of those reported from the British Isles. ${ }^{191213}$ Other studies of HSES describe case reports or small series. It is therefore important to compare some features of our study with reports by Bacon and Hall from the British Isles. ${ }^{9}$ Seasonality, with peak incidence in winter and early spring was a clear finding in our area. It was less prominent in the British Isles, although $56 \%$ of cases were admitted in the winter season, usually January/February. The infants' age in our study ranged between 6 and 32 weeks; median 14 weeks, which is comparable with a median of 15 weeks reported in the British study. ${ }^{9}$

Raised body temperature was indicated as a mandatory criterion for the establishment of HSES. $^{3}$ Three of our patients, and seven reported by Bacon and $\mathrm{Hall}^{9}{ }^{9}$ did not have a raised body temperature on arrival at the emergency room. All three of our patients however felt hot, according to the parents, before arrival at the emergency room, and two of three infants had documented higher body temperatures shortly before arrival. As noted by Bacon and Hall, their patients had been stripped for some time before recording body temperature. Furthermore, all patients arrived at the emergency room in shock. This may explain the abrupt drop in body temperature documented in our patients between emer- gency room arrival (mean $39.4^{\circ} \mathrm{C}$ ) and the PICU (mean $36.05^{\circ} \mathrm{C}$ ). This also suggests that body temperature for most infants had peaked before arrival. In this regard, it is important to compare the clustering of admission times (5.00 am to $9.00 \mathrm{am}$ ) in our study with those reported by Bacon and Hall (8.00 am to 11.00 am), which supports the notion that hyperpyrexia started and peaked at night when the infant is particularly at risk from heat injury. ${ }^{22}$

In summary, our data support previous suggestions that some environmental manipulations or other physiological stress, for example common viral infection, in a genetically determined, susceptible individual may cause hyperpyrexia resulting in HSES with multiple organ failure.

The folklore that infants with fever should be wrapped up well and kept warm may be dangerous for some babies. Physicians, nurses, and other health professionals should inform parents that mildly ill infants do not have to be kept excessively warm.

1 Levin M, Kay JD, Gould JD, et al. Hemorrhagic shock and encephalopathy: a new syndrome with a high mortality in young children. Lancet 1983; ii: 64-7.

2 Whittington LK, Roscelli JD, Parry WH. Hemorrhagic shock and encephalopathy: further description of a new syndrome. F Pediatr 1985; 106: 599-602.

3 Chaves-Carballo E, Monets JE, Nelson B, Chrenka BA. Hemorrhagic shock and encephalopathy. Clinical definition of a catastrophic syndrome in infants. Am $\mathcal{f}$ Dis Child 1990; 144: 1079-82

4 Sofer S, Philip M, Hershkowits J, Bennett H. Hemorrhagic shock and encephalopathy syndrome. Its association with hyperthermia. Am $\mathcal{F}$ Dis Child 1986; 140: 1252-4.

5 Van Acker KJ, Roodhooft AM, Van Bever H. Hemorrhagic shock and encephalopathy. Eur $\mathcal{F}$ Pediatr 1986; 145: 66-9.

6 Nelson EAS, Taylor BJ, Dempster AG. Hemorrhagic shock encephalopathy syndrome. $N Z$ Med f 1988; 101: 69-71.

7 Tam AYC, Ng IOL, Cheng PT, Tang TS, Li CH. Hemorrhagic shock encephalopathy. Acta Paediatr Scand 1989; 78: 458-62.

8 Chiu NC, Shen EY, Lee HC. Hemorrhagic shock encephalopathy syndrome: report of two cases. Acta Paediatr Sin 1989; 30: 118-21.

9 Bacon CJ, Hall SM. Haemorrhagic shock encephalopathy syndrome in the British Isles. Arch Dis Child 1992; 67: 98593.

10 Sofer S. Hemorrhagic shock and encephalopathy: a new syndrome in young children associated with high morbidity and mortality. Harefuah 1988; 115: 63-6.

11 Sofer S, Shahak E. Myocardial infarction in hemorrhagic shock and encephalopathy syndrome. Pediatr Emerg Care 1989; 5: 99-101.

12 Report of Communicable Disease Surveillance Centre, UK. Joint British Paediatric Association and Communicable Diseases Surveillance Centre. Surveillance scheme for haemorrhagic shock encephalopathy syndrome: surveilhaemorrhagic shock encephalopathy syndrome:

13 Levin M, Pincott JR, Hjelm M, et al. Hemorrhagic shock and encephalopathy: clinical, pathologic, and biochemical features. $\mathcal{F}$ Pediatr 1989; 114: 194-203.

14 Ball WS Jr, Prenger EC. Skull and contents. In: Kirks DR, ed. 2nd Ed. Practical pediatric imaging. Diagnostic radiology of infants and children. Boston: Little Brown and Company, 1991:165-66.

15 Zuckerman GB, Conway EE, Singer L. Hemorrhagic shock and encephalopathy syndrome and heatstroke: a physiologic comparison of two entities. Pediatr Emerg Care 1994; 10: 172-7.

16 Katz M, Holcberg G, Glezerman M, et al. Incidence of congenital malformations in the Bedouin population of the Negev. Isr f Obstet Gynecol 1990; 1: 175-8.

17 Potashnic R, Carmi R, Sofer S, Bashan N, Abeliovich D. Maple syrup urine disease in a Bedouin tribe. Pre and Maple syrup urine disease in a Bedouin tribe.

18 Sofer S, Shahak E, Gueron M. Scorpion envenomation and antivenom therapy. $\mathcal{F}$ Pediatr 1994; 124: 973-8.

19 Sofer S, Tal A, Shahak E. Carbamate and organophosphate poisoning in early childhood. Pediatr Emerg Care 1989; 5: 222-5.

20 Sofer S, Yagupsky P, Hershkowits J, Bearman JE. Improved outcome of hypothermic infants. Pediatr Emerg Care 1986; 2: $211-4$.

21 Eiser C, Town C, Tripp J. Dress and care of infants in health and illness. Arch Dis Child 1985; 60: 465-70.

22 Bacon CJ, Bell SA, Clulow EE, Beattie AB. How mothers keep their babies warm. Arch Dis Child 1991; 66: 627-32. 